\title{
Cold Atomic Gases in Optical Lattices with Disorder
}

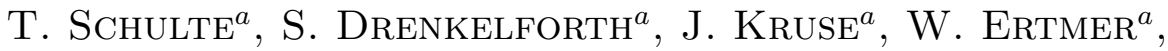 \\ J.J. Arlt ${ }^{a}$, A. KAntian ${ }^{b, c}$, L. SAnChez-PAlencia ${ }^{b, d}$,

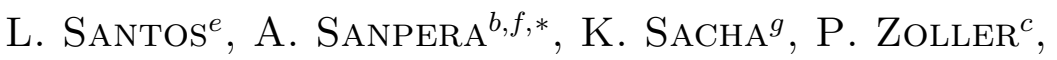 \\ M. LEWENSTEIN ${ }^{b, h, *}$ AND J. ZAKRZEWSKI ${ }^{g}$ \\ ${ }^{a}$ Institut für Quantenoptik, Universität Hannover \\ 30167 Hannover, Germany \\ ${ }^{b}$ Institut für Theoretische Physik, Universität Hannover \\ 30167 Hannover, Germany \\ ${ }^{c}$ Institute of Theoretical Physics, Universität Innsbruck \\ Technikerstr. 25, 6020 Innsbruck, Austria \\ ${ }^{d}$ Laboratoire Charles Fabry, Institut d'Optique \\ Université Paris-Sud XI, 91403 Orsay cedex, France \\ ${ }^{e}$ Institut für Theoretische Physik III \\ Pfaffenwaldring 57, 70550 Stuttgart, Germany \\ ${ }^{f}$ Department of Physics, Theoretical Physics Group \\ Universidas Autonoma Barcelona, 08193 Bellaterra, Spain \\ ${ }^{g}$ Instytut Fizyki Mariana Smoluchowskiego \\ Uniwersytet Jagielloński, 30-059 Kraków, Poland \\ ${ }^{h}$ Institut de Ciències Fotòniques, 08034 Barcelona, Spain
}

Cold atomic gases placed in optical lattices enable studies of simple condensed matter theory models with parameters that may be tuned relatively easily. When the optical potential is randomized (e.g. using laser speckle to create a random intensity distribution) one may be able to observe Anderson localization of matter waves for non-interacting bosons, the so-called Bose glass in the presence of interactions, as well as the Fermi glass or quantum spin glass for mixtures of fermions and bosons.

PACS numbers: 03.75.Kk, 03.75.Lm, 05.30.Jp, 64.60.Cn

*Also at Institució Catalana de Recerca i Estudis Avançats. 


\section{Introduction}

Cold atoms placed in an optical lattice potential make it possible to realize standard models of condensed matter theory as suggested [1] for the Bose-Hubbard $(\mathrm{BH})$ model $[2,3]$. The reported realization [4] of a quantum phase transition between superfluid (SF) and Mott insulator (MI) phases showed convincingly that it was possible to experimentally control the parameters of the model practically at will. This triggered several studies involving Bose condensates [5-9] as well as more recently Bose-Fermi (BF) mixtures [10-13] placed in an optical lattice. The latter have no direct analogues in condensed matter physics, and provide an exciting possibility of boson-fermion pairing [13] and of creating novel phases [12, 14].

It is natural to consider whether the presence of disorder may affect the properties of these atomic systems. For the well studied $\mathrm{BH}$ model the answer is known since the seminal study of late eighties [2]. In the absence of disorder there exist MI states, characterized by a fixed (integer) number of bosons per lattice site, a gap in the excitation spectrum, and vanishing superfluid fraction and compressibility. An additional insulating phase, the so-called Bose-glass (BG) phase may occur in the presence of disorder [2]. The BG is characterized by the absence of the superfluid fraction, a gapless excitation spectrum, and a finite compressibility.

The nature of disorder-induced insulator phases depends on the interplay between hopping, nonlinear interactions and disorder. In the strong-interaction regime, the cooperation of interactions and disorder leads to the appearance of a BG phase. For weak interactions, the situation is rather different. Here the interactions tend to delocalize and therefore compete with the disorder (which, for no interactions, may lead to the Anderson localization) sometimes destroying the localization altogether while for other conditions allowing for the formation of an Anderson-type insulator, or the Anderson glass (AG) [15]. Here no superfluid fraction is present, while the condensate fraction is large.

It still remains to be seen whether the richness of disorder induced phenomena may be observed experimentally in typical cold atoms setups. This contribution aims to give a partial answer to this question. We briefly mention three different cases in the subsequent sections: Anderson-like localization for a one-dimensional lattice with disorder, MI-BG transition for deep lattices, and BF mixtures.

\section{Anderson limit}

Let us consider first a typical arrangement of a Bose condensate placed in an elongated harmonic trap as realized in Florence [16] or Hannover [17]. In such a trap the condensate may be treated as quasi one-dimensional (1D). In the Florence experiment the condensate is placed in a random optical potential resulting from the projection of speckle light. The Bose-Einstein condensate (BEC) probes about 6 (random) wells in the axial direction. One then expects that the BEC 
splits between the wells, and that the wells size bounds from above the BEC phase coherence length; detection based on the time of flight (TOF) expansion will then reveal stripes in the condensate image, caused by the transformation of the phase fluctuations to the density modulations, similar to that for highly elongated condensates $[18,19]$. We speculate that the spacing between stripes observed in the experiment [16], seemingly in disagreement with the characteristic length of the speckle, may in fact be related to them if the interactions during the initial stage of TOF expansion is taken into account.

In the Hannover experiment a speckle of similar characteristic length $L$ is applied together with a $1 \mathrm{D}$ optical lattice with $\lambda=825 \mathrm{~nm}$. Preliminary observations confirm the presence of the stripes observed in Florence; due to the presence of the lattice striped Bragg side bands appear in addition. Let us note, however, that since $\lambda \ll L$ the speckle produces a smooth modulation of the optical potential.

To check whether one can observe the Anderson localization in the presence of interactions in realistic experimental conditions we assume parameters similar to those of the Hannover experiment. We find the ground state of the 1D GrossPitaevskii equation (GPE) which should be appropriate for quasi-1D experimental situation ${ }^{\dagger}$.

In oscillator units corresponding to the axial trap frequency the time-independent GPE reads

$$
\left[-\frac{\partial_{x}^{2}}{2}+\frac{x^{2}}{2}+V_{0} \cos ^{2}(k x)+V_{\mathrm{dis}}(x)+g|\phi|^{2}\right] \phi=\mu \phi,
$$

where $V_{0}$ is the amplitude of the optical lattice created by $\lambda=825 \mathrm{~nm}$ laser beam with $k=2 \pi / \lambda$. We take $V_{0}=6.5 E_{\mathrm{r}}$, where $E_{\mathrm{r}}$ is the recoil energy. $V_{\mathrm{dis}}(x)$ stands for a disorder potential. The interaction coefficient $g$ is chosen such that the axial size of the BEC corresponds to that of the (necessarily three-dimensional) experiment [17].

While the details of the simulations will be presented elsewhere, let us mention here the main message. As mentioned above, no nontrivial localization is expected for large speckles with a correlation length order of magnitude bigger than $\lambda$. One can decrease $L$ so it is of the order of $\lambda$ in numerical simulations (albeit it may be difficult to realize experimentally). Still, for realistic experimental situations (minimal number of atoms of the order of $10^{4}$ — that determines the size of the BEC and the effective interaction strength $g$ ) no localization is present due to the relatively strong interactions that tend to destroy the localization. The effect can be easily understood considering the effective potential seen by the atoms

\footnotetext{
${ }^{\dagger}$ Actually we have checked, using second order Bogoliubov expansion in selected cases that, for the values of parameters studied, the depletion of the condensate is of the order of few percent, so the mean field GPE description should be well justified.
} 


$$
V_{\mathrm{eff}}=\frac{x^{2}}{2}+V_{0} \cos ^{2}(k x)+V_{\mathrm{dis}}(x)+g|\phi|^{2} .
$$

Localization appears for $g=0$ due to the random nature of $V_{\text {dis }}(x)$. The nonlinear term effectively screens the disorder, with $g|\phi|^{2}$ being large at the minima of the optical potential.

To observe localization one needs thus also to decrease the interaction strength $g$. Several methods are possible, e.g. by modifying the interactions via a Feshbach resonance technique. One may also consider a smaller number of atoms - but then the BEC typically has a much smaller Thomas-Fermi radius and occupies few wells of the optical potential. Another possibility is to make the harmonic trap frequency much smaller - then the BEC spreads more and a smaller number of atoms may be used. Figure 1 shows the ground state for a noninteracting system as well as for a small value of $g$ corresponding to a very shallow trap. Actually instead of small-size speckles we create an effective disorder by superimposing two additional laser beams with incommensurate frequencies $[1,9]$.

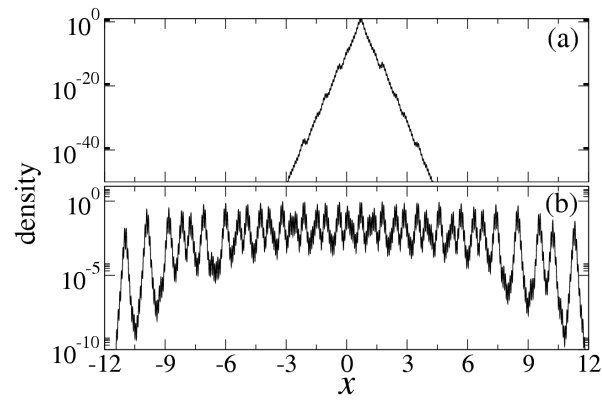

Fig. 1. Ground state of BEC in an optical lattice with superimposed disorder. The disorder is created by additional optical lattices (30 times weaker than the main optical lattice). Part (a) corresponds to no interactions $(g=0)$, part (b) to $g=128$ corresponding to 5000 atoms in a shallow trap with a frequency around $4 \mathrm{~Hz}$.

Let us mention that similar destruction of localization has been predicted by Shepelyansky [20] in the case of dynamical localization perturbed by a weak nonlinearity. Similarly, effective screening of the disorder potential by interacting bosons has been considered before (see [21] and references therein). The nice feature here is that these problems may now also be addressed in the cold atoms environment. Moreover, our simulations show the details of onset of the screening effect (see Fig. 1b), which consists in appearance in the condensate wave function of many exponentially localized peaks of comparable amplitudes.

\section{Bose-Hubbard model considerations}

Up to now we have considered a 1D situation where several atoms occupy a single well of the optical lattice. Without disorder we are in the deep superfluid 
regime (this justifies the application of the GPE). For higher dimensional lattices we may expect fewer atoms per site even when the total number of atoms exceeds $10^{5}$ (e.g. in $3 \mathrm{D}$ case of [4] up to 3 atoms per site is expected). Then there is a chance to reach an insulating regime.

For a sufficiently high optical lattice, atoms occupy the lowest band of the lattice. A convenient description of the system (with no disorder) in that case is via the tight-binding Bose-Hubbard model [1] with the Hamiltonian

$$
H=-\sum_{\langle i, j\rangle} J b_{i}^{\dagger} b_{j}+\frac{U}{2} \sum_{i} n_{i}\left(n_{i}-1\right)+\sum_{i} W_{i} n_{i},
$$

with $b_{i}$ annihilating a boson at site $i, J$ being the tunneling rate between nearest neighbors (nn), while $U$ being the effective interaction between atoms in the same well. $W_{i}$ is the energy of the bottom of $i$-th well, which depends on the trapping potential, and typically will be assumed to be harmonic. Let us superimpose the disorder on top of that lattice, either by adding additional lattices or by adding the speckle. This modifies $W_{i}$ and, for a sufficiently strong disorder also the tunneling coefficients which receive an additional random component. For a weak disorder the main effect comes from modifying $W_{i}$.

Following [9] we consider a 2D lattice (assuming a tight harmonic binding in the perpendicular direction) and neglect the harmonic binding. To be specific we consider sodium atoms and optical lattice created by $\lambda=1064 \mathrm{~nm}$ radiation. The optical lattice potential takes the form

$$
V_{l}(\boldsymbol{r})=V_{0}\left[\cos ^{2}(k x)+\cos ^{2}(k y)\right]
$$

with $k=2 \pi / \lambda$. When tunneling dominates (small $U / J$ ) the ground state is superfluid. With the increase in the lattice height a phase transition to the Mott insulator phase may occur if the ratio of the number of atoms $N$ to number of sites $M, \kappa=N / M$ is commensurate. For a non-integer $\kappa$ the fraction of atoms will remain always superfluid. We expect then that when disorder is adiabatically turned on the system will undergo a phase transition to the Bose glass phase i.e. the superfluid fraction will vanish.

The exact study of the dynamics, for a 2D lattice is impossible due to computer memory limitations. Therefore, we use the dynamical mean field approach, which neglects the entanglement between different wells and expresses the wave function as a product of states at different wells. This is the so-called Gutzwiller ansatz $\left|\Psi_{\mathrm{MF}}\right\rangle=\prod_{i} \sum_{n}^{\infty} f_{n}^{(i)}|n\rangle_{i}$, where the $f_{n}^{(i)}$ are the amplitudes of having $n$ atoms at an $i$-th lattice site. The adiabatic changes of the ground state when parameters of the system are varied may then be studied using the dynamical Gutzwiller approach [22], see [9] for details. Such an approach has been recently used by one of us [23] for simulating details of the experiment on MI-SF quantum phase transition [4].

To characterize the system one may use the so-called condensate fraction, defined as the highest eigenvalue of the one particle density matrix, $\rho_{i j}=$ 
$\left\langle\Psi_{\mathrm{MF}}\left|b_{i}^{\dagger} b_{j}\right| \Psi_{\mathrm{MF}}\right\rangle$, divided by the number of particles [24]. Another useful quantity is the superfluid fraction which is determined by quantifying systems sensitivity to changes of boundary conditions using the so-called "boost method" (see [25, 26] for details).

To simulate the transition from a superfluid to a Bose glass one should consider first a BEC, then turn on optical lattices to obtain a SF state, then turn on the disorder. The BH model as a tight binding model assumes that the optical lattice is sufficiently deep. To stay within the model we assume that initially atoms are in a SF ground state with $V_{0}=V_{\mathrm{SF}}=7 E_{\mathrm{r}}$ (this and the other values for parameters are stimulated by experiments [4]). Then we increase the lattice depth using a linear ramp $V_{0}(t)=V_{\mathrm{SF}}+\left(V_{\mathrm{MI}}-V_{\mathrm{SF}}\right) t / T$ with $T=20 \mathrm{~ms}$ and $V_{\mathrm{MI}}=25 E_{\mathrm{r}}$. For integer $\kappa$ we should reach then a pure MI state. Instead we take $\kappa=0.75$ so in the process the superfluid fraction decreases from almost unity to about $30 \%$. With no disorder the condensate fraction remains equal to the superfluid fraction.

Next we introduce disorder. To this end we employ an additional 2D lattice with $\lambda=795 \mathrm{~nm}$ and main axes rotated with respect to the original lattice. The amplitude of that additional lattice is turned on very slowly (over more than $400 \mathrm{~ms}$ ) with the linear ramp finally reaching $V_{1}=0.059 E_{\mathrm{r}}$. Let us note that this final value is only a tiny fraction of the amplitude of the main lattice. Still the decrease in the SF fraction (and the condensate fraction) is quite spectacular (cf. Fig. 2). The condensate fraction stays at a few percent level, while the SF fraction practically vanishes.

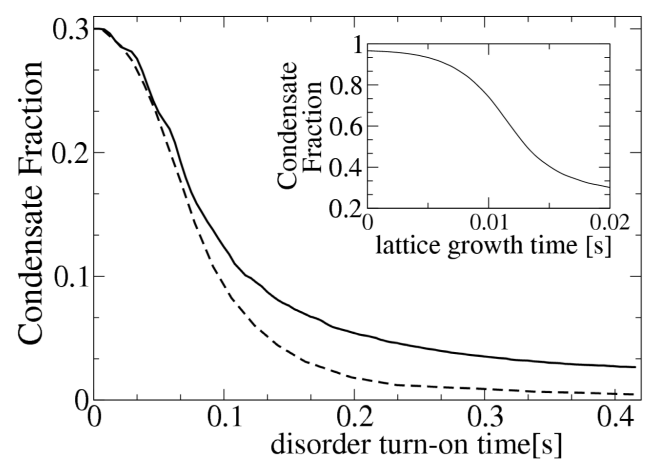

Fig. 2. Condensate fraction (solid line) during the dynamical SF to BG transition induced by a disorder created by an additional optical lattice, for ${ }^{23} \mathrm{Na}$ atoms placed on the main $40 \times 40$ lattice with $\lambda=1064 \mathrm{~nm}, \kappa=0.75$. The inset shows the first preparation step (see text). The dashed line presents the SF fraction.

This result seems quite promising. It seems that $\mathrm{SF}-\mathrm{BG}$ transition may be observed in cold atoms experiments. Still one should be aware of the difficulties. To follow the ground state adiabatically, the disorder must be turned on quite slowly. Let us observe that the turn on time above was a fraction of a second. 
This is due to the fact that the presence of the disorder leads to creation of a manifold of quasidegenerate states - to be adiabatic takes a lot of time. That may create difficulties with decoherence as well as other mechanisms which may affect the experiment.

\section{Bose-Fermi mixtures}

Let us now consider briefly the effect of disorder on Bose-Fermi mixtures placed in optical lattices. Here, instead of dynamics, we merely indicate the richness of possible behaviors by considering the properties of the ground state within the effective Hamiltonian approach [27].

Under appropriate conditions Bose-Fermi mixtures can be well described by the Bose-Fermi-Hubbard (BFH) model [11]. A particularly appealing feature of this model is the possibility to produce novel quantum phases $[12,14]$, fermion-boson induced superfluidity [28], and composite fermions, which for attractive (repulsive) interactions between fermions and bosons, are formed by a fermion and bosons (bosonic holes) [13, 29,30]. The phase diagram of the system has been determined in Ref. [31] by means of the mean-field theory [3]. These studies have been generalized to inhomogeneous lattices [32] to include simultaneously the effects of the lattice and of a possible trap potential. Treating the tunneling as a perturbation one may also include the effect of the disorder [27].

Let us consider a sample of ultracold bosonic and (polarized) fermionic atoms (e.g. ${ }^{7} \mathrm{Li}^{6}{ }^{6} \mathrm{Li}$ or ${ }^{87} \mathrm{Rb}^{40} \mathrm{~K}$ ) trapped in an inhomogeneous optical lattice. We assume that the lattice potential consists of two terms: a periodic potential forming a hypercubic lattice in $2 \mathrm{D}$ or $3 \mathrm{D}$, and an additional, e.g. disordered superlattice potential. The atoms occupy only the lowest energy band, i.e. the fermion number $N_{\mathrm{F}}$ is strictly smaller than the number of lattice sites $M$. The Hamiltonian of the system reads [11, 33]:

$$
\begin{aligned}
& H_{\mathrm{BFH}}=-\sum_{\langle i j\rangle}\left(J_{\mathrm{B}} b_{i}^{\dagger} b_{j}+J_{\mathrm{F}} f_{i}^{\dagger} f_{j}+\text { h.c. }\right) \\
& +\sum_{i}\left[\frac{1}{2} U n_{i}\left(n_{i}-1\right)+V n_{i} m_{i}-\mu_{i}^{\mathrm{B}} n_{i}-\mu_{i}^{\mathrm{F}} m_{i}\right],
\end{aligned}
$$

where $b_{i}\left(f_{i}\right)$ annihilates a boson (fermion) at site $i, n_{i}=b_{i}^{\dagger} b_{i}, m_{i}=f_{i}^{\dagger} f_{i}$, and $\mu_{i}^{\mathrm{B}}$ and $\mu_{i}^{\mathrm{F}}$ are the bosonic and fermionic local chemical potentials, respectively. Later on we assume the same tunneling rate for fermions and bosons, $J_{\mathrm{B}}=J_{\mathrm{F}}=J$, and consider repulsive boson-boson interactions $U$, as well as repulsive on-site bosonfermion interactions $V$. Since we want to treat tunneling perturbatively, we assume strong interactions: $V, U \gg J$, and introduce a shorthand notation $\alpha=U / V>0$. We assume that disorder affects only bosons, so we take $[27] \mu_{i}^{\mathrm{F}}=0, \mu_{i}^{\mathrm{B}}=\mu_{i} U$.

Let us consider first $J=0$. For $0 \leq \mu_{i}<1$ (the situation discussed here) in the absence of a fermion one expects one boson per site. The fermions tend to push bosons out of different sites. In fact there are two possibilities: 
(i) $A$-sites, for which $\mu_{i}-\alpha \geq 0$, and these fermions do not push bosons out, and (ii) $B$-sites, for which $\mu_{i}-\alpha<0$, and the fermion pushes the boson out forming a composite fermion-bosonic hole. Energetically, the second situation is favorable, so the fermions tend first to fill $B$-sites and only if $N_{\mathrm{F}}>N_{B}$ they will start to occupy the $A$-sites. The number of $A(B)$ sites $N_{A}\left(N_{B}\right)$ depends on $\mu_{i}$ 's as well as the presence of a possible additional harmonic trap. We construct the corresponding projector operators $P, Q=1-P$, which depend on $N_{A}$ and $N_{\mathrm{F}}$. Using second order effective Hamiltonian approach [34] we obtain $H_{\text {eff }}$ in the form

$$
H_{\mathrm{eff}}=\sum_{\langle i j\rangle}\left[-\left(d_{i j} F_{i}^{\dagger} F_{j}+\text { h.c. }\right)+K_{i j} M_{i} M_{j}\right]+\sum_{i} \tilde{\mu}_{i} M_{i} .
$$

Now $F_{i}, F_{i}^{\dagger}$ are the corresponding composite fermionic annihilation and creation operators, and $M_{i}=F_{i}^{\dagger} F_{i}$. The hopping amplitudes $d_{i j}$ and the nn couplings $K_{i j}$ (which might be repulsive $(>0)$ or attractive $(<0)$ ) are of order of $J^{2} / U$. The hopping $i \rightarrow j$, or back causes the energy change $\pm \Delta_{i j}=\mu_{i}-\mu_{j}$ in units of $U$, i.e. is highly non-resonant and inefficient for $\Delta_{i j} \simeq 1$. Composite fermions may feel the local disordered chemical potential $\tilde{\mu}_{i}$.

We briefly mention here different physical cases, while more details may be found in Ref. [27]. We consider the situation when all sites are of type $B$. We have then a gas of composites flowing within the MI with 1 boson per site. The following interesting cases may be realized:

- We may have attractive (although random) interactions for $\alpha>1, K_{i j} \leq 0$.

- The qualitative character of interactions may be controlled from site to site for $\alpha \leq 1$, since $K_{i j}$ may in these cases take positive or negative values for $\Delta_{i j}$ small or $\Delta_{i j} \simeq \alpha$.

Let us consider a small disorder; then one may neglect the site-dependence of $d_{i j}$ and $K_{i j}$ denoting them as $d$ and $K$, respectively. It is then feasible to realize the following quantum phases:

- Fermi-glass phase is realized for $K \ll d$. The states are Anderson-like localized, although include many-body effects, which may tend to delocalize the system - see the case of bosons discussed above.

- For repulsive interactions and $K / d \gg 1$, the ground state will be a Mott insulator for large enough and commensurate filling factors. The composite fermions will be pinned. In particular for $1 / 2$ filling the so-called checkerboard phase is expected.

- $\Delta_{i j} \simeq \alpha \simeq 1$. The tunneling becomes then non-resonant and negligible, while the couplings $K_{i j}$ fluctuate strongly. The (fermionic) Ising spin glass model is realized [35] described by the Edwards-Anderson model with $s_{i}=$ $2 M_{i}-1= \pm 1[36]$ : 


$$
H_{\mathrm{EA}}=\frac{1}{4} \sum_{\langle i j\rangle} K_{i j} s_{i} s_{j}+\sum_{i} \tilde{\mu}_{i} s_{i} / 2 .
$$

Let us note that the last case suggests to address various open problems of spin-glass theory via cold atoms experiments! Let us mention that in the presence of $A$-sites we can address the different questions concerning quantum percolation in the model, too [27].

\section{Summary}

We have discussed how disorder may lead to modifications of the physics of cold atoms placed in optical lattices. We have barely touched the subject, the interested reader is referred to original papers. We point out the difficulties which arrive when a reaL experiment is being planned. On the other hand, it may be worthwhile to attempt to overcome possible difficulties - model studies indicate that several situations known from condensed matter theory as well as novel ones may be addressed via cold atoms physics.

Damski has made a major contribution to the results reviewed here. Meanwhile, he has developed several novel interesting ideas [37, 38], related to physics of ultracold disordered gases, involving frustrated systems without disorder, and quenching in the dynamics of quantum phase transitions. It is also worth mentioning recent work of two of us on ultracold atomic gases in quasi-crystal lattices [39].

\section{Acknowledgments}

We acknowledge support from the Deutsche Forschungsgemeinschaft (SFB 407 and SPP1116), the RTN Cold Quantum Gases, ESF PESC BEC2000+, the Alexander von Humboldt Foundation. This work was also supported by Polish government Scientific funds PBZ-MIN-008/P03/2003 (J.Z.) and 1P03B08328 (2005-08) (K.S.).

\section{Note added in proof}

After submittal of this paper, we became aware of recent work in the Orsay group where suppression of transport in the elongated BEC in a random potential has been observed [40]. No additional optical lattice potential is present in the Orsay experiment in difference to the case discussed in the present paper. Similarly, no additional lattice is present in the recent Florence experiment [41]. Both [40] and [41] do not observe Anderson localization.

\section{References}

[1] D. Jaksch, C. Bruder, J.I. Cirac, C.W. Gardiner, P. Zoller, Phys. Rev. Lett. 81, 3108 (1998).

[2] M.P.A. Fisher, P.B. Weichman, G. Grinstein, D.S. Fisher, Phys. Rev. B 40, 546 (1989). 
[3] S. Sachdev, Quantum Phase Transitions, Cambridge University Press, Cambridge 1999.

[4] M. Greiner, O. Mandel, T. Esslinger, T.W. Hämsch, I. Bloch, Nature 415, 39 (2002).

[5] D. van Oosten, P. van der Straten, H.T.C. Stoof, Phys. Rev. A 63, 053601 (2001).

[6] D.B.M. Dickerscheid, D. van Oosten, P.J.H. Denteneer, H.T.C. Stoof, Phys. Rev. A 68, 043623 (2003).

[7] C. Menotti, A. Smerzi, A. Trombettoni, New J. Phys. 5, 112 (2003).

[8] B. Damski, L. Santos, E. Tiemann, M. Lewenstein, S. Kotochigova, P. Julienne, P. Zoller, Phys. Rev. Lett. 90, 110401 (2003).

[9] B. Damski, J. Zakrzewski, L. Santos, P. Zoller, M. Lewenstein, Phys. Rev. Lett. 91, 080403 (2003).

[10] G. Modugno, F. Ferlaino, R. Heidemann, G. Roati, M. Inguscio, Phys. Rev. A 68, 011601(R) (2003).

[11] A. Albus, F. Illuminati, J. Eisert, Phys. Rev. A 68, 023606 (2003).

[12] H.P. Büchler, G. Blatter, Phys. Rev. Lett. 91, 130404 (2003).

[13] M. Lewenstein, L. Santos, M. Baranov, H. Fehrmann, Phys. Rev. Lett. 92, 050401 (2004).

[14] R. Roth, K. Burnettt, Phys. Rev. A 68, 023604 (2003).

[15] G.G. Batrouni, R.T. Scalettar, G.T. Zimanyi, Phys. Rev. Lett. 65, 1765 (1990); R.T. Scalettar, G.G. Batrouni, G.T. Zimanyi, ibid. 66, 3144 (1991).

[16] J.E. Lye, L. Fallani, M. Modugno, D. Wiersma, C. Fort, M. Inguscio, Phys. Rev. Lett. 95, 070401 (2005).

[17] T. Schulte, S. Drenkelforth, J. Kruse, W. Ertmer, J. Arlt, K. Sacha, J. Zakrzewski, M. Lewenstein, Phys. Rev. Lett. 95, 170411 (2005).

[18] S. Dettmer, D. Hellweg, P. Ryytty, J.J. Arlt, W. Ertmer, K. Sengstock, D.S. Petrov, G.V. Shlyapnikov, H. Kreutzmann, L. Santos, M. Lewenstein, Phys. Rev. Lett. 87, 160406 (2001).

[19] S. Richard, F. Gerbier, J.H. Thywissen, M. Hugbart, P. Bouyer, A. Aspect, Phys. Rev. Lett. 91, 010405 (2003).

[20] D.L. Shepelyansky, Phys. Rev. Lett. 70, 1787 (1993).

[21] K.G. Singh, D.S. Rokhsar, Phys. Rev. B 49, 9013 (1994).

[22] D. Jaksch, V. Venturi, J.I. Cirac, C.J. Williams, P. Zoller, Phys. Rev. Lett. 89, 040402 (2002).

[23] J. Zakrzewski, Phys. Rev. A 71, 043601 (2005); Erratum, Phys. Rev. A 72, 039904 (2005).

[24] F. Dalfovo, S. Giorgini, L.P. Pitaevskii, S. Stringari, Rev. Mod. Phys. 71, 463 (1999).

[25] E.H. Lieb, R. Seiringer, J. Yngvason, Phys. Rev. B 66, 134529 (2002).

[26] R. Roth, K. Burnett, Phys. Rev. A 67, 031602(R) (2003).

[27] A. Sanpera, A. Kantian, L. Sanchez-Palencia, J. Zakrzewski, M. Lewenstein, Phys. Rev. Lett. 93, 040401 (2004). 
[28] F. Illuminati, A. Albus, Phys. Rev. Lett. 93, 090406 (2004).

[29] A.B. Kuklov, B.V. Svistunov, Phys. Rev. Lett. 90, 100401 (2003).

[30] M.Yu. Kagan, I.V. Brodsky, D.V. Efremov, A.V. Klaptsov, cond-mat/0209481.

[31] H. Fehrmann, M.A. Baranov, B. Damski, M. Lewenstein, L. Santos, Opt. Commun. 243, 23 (2004).

[32] M. Cramer, J. Eisert, F. Illuminati, Phys. Rev. Lett. 93, 190405 (2004).

[33] A. Auerbach, Interacting Electrons and Quantum Magnetism, Springer, New York 1994.

[34] C. Cohen-Tannoudji, J. Dupont-Roc, G. Grynberg, Atom-Photon Interactions: Basic Processes and Applications, Wiley, New York 1992.

[35] R. Oppermann, B. Rosenow, Phys. Rev. B 60, 10325 (1999).

[36] M. Mézard, G. Parisi, M.A. Virasoro, Spin Glass and Beyond, World Scientific, Singapore 1987.

[37] B. Damski, H.-U. Everts, A. Honecker, H. Fehrmann, L. Santos, M. Lewenstein, Phys. Rev. A 72, 053612 (2005).

[38] B. Damski, cond-mat/0411004, Phys. Rev. Lett., in press.

[39] L. Sanchez-Palencia, L. Santos, Phys. Rev. A 72, 053607 (2005).

[40] D. Clément, A.F. Varon, M. Hugbart, J.A. Retter, P. Bouyer, L. Sanchez-Palencia, D.M. Gangardt, G.V. Shlyapnikov, A. Aspect, Phys. Rev. Lett. 95, 170409 (2005).

[41] C. Fort, L. Fallani, V. Guarrera, J. Lye, M. Modugno, D.S. Wiersma, M. Inguscio, Phys. Rev. Lett. 95, 170410 (2005). 